\title{
Autoimmune Encephalitis in Children: A Short Review
}

\author{
Shubhankar Mishra* \\ Senior resident, Department of Neurology, SCB Medical College, India
}

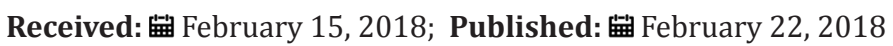

*Corresponding author: Shubhankar Mishra, Senior resident, Department of Neurology, SCB Medical College, Cuttack, Odisha, India

\begin{abstract}
Autoimmune encephalitis (AE) comprises an expanding group of clinical syndromes that can occur at all ages, but preferentially affect younger adults and children. These disorders associate with antibodies against neuronal cell surface proteins and synaptic receptors involved in synaptic transmission, plasticity, or neuronal excitability. The spectrums of symptoms include psychosis, catatonia, alterations of behaviour and memory, seizures, abnormal movements, and autonomic dysregulations. Inflammatory findings in the cerebrospinal fluid may be present. Magnetic resonance imaging (MRI) may also demonstrate abnormalities for better diagnosis, particularly on fluidattenuated inversion recovery or T2-weighted images. Patients may initially be diagnosed with idiopathic encephalitis, likely viral but with negative viral studies. Autoimmune encephalitis should be included in the differential diagnosis of any patient, especially if young, with a rapidly progressive encephalopathy of unclear origin. AE is well responsive to immune therapy, with prompt diagnosis and treatment strongly beneficial.
\end{abstract}

Keywords: Autoimmune encephalitis; NMDA; Steroids

Abbreviations: AE: Autoimmune encephalitis; MRI: Magnetic Resonance Imaging

\section{Introduction}

Encephalitis refers to an inflammatory disorder of the brain causing disastrous symptomatologies. The causes of encephalitis are numerous but finding a causative agent is always a difficult task. A study by the California Encephalitis Project, a centre focused in the epidemiology and aetiology of encephalitis, found that $63 \%$ of the patients remained without an aetiology after a battery of tests for 16 potential infectious agents [1]. Recently many auto antibodies are discovered who target specific antigens in the CNS and cause features of clinical encephalitis [2]. The immunological trigger of autoimmune encephalitis is varied and in many cases yet to be established. In some patients the presence of a systemic tumour that expresses the target neuronal/synaptic proteins appears to be important, but it's more common in adult age groups. Any immunological type of autoimmune encephalitis can have a relapsing course and therefore the diagnosis of these disorders should be considered in patients with a past history of encephalitis or relapsing encephalopathy [3-8] (Table 1).

Table 1: classification, mechanism and salient features of autoimmune encephalopathy.

\begin{tabular}{|c|c|c|c|c|}
\hline & Mechanism & Features & Diagnosis & Treatment \\
\hline \multicolumn{5}{|c|}{ Demonstrated immune mechanism } \\
\hline $\begin{array}{l}\text { Anti NMDAR Immune } \\
\text { mechanism }^{3}\end{array}$ & $\begin{array}{l}\text { Ab againstNR1 subunit } \\
\text { of NMDAR. } \\
\text { Disrupt function by } \\
\text { cross linking and } \\
\text { internalization of } \\
\text { receptors }\end{array}$ & $\begin{array}{l}\text { Psychosis, seizures, } \\
\text { dyskinesia, autonomic } \\
\text { dysfunction }^{4}\end{array}$ & $\begin{array}{c}\text { EEG: abnormal; } \\
\text { "extreme delta brush" pattern }{ }^{6} \\
\text { MRI: nonspecific } \\
\text { findings } \\
\text { CSF: pleocytosis and/or increased } \\
\text { protein in }>80 \%\end{array}$ & $\begin{array}{l}\text { Immunotherapy } \\
\text { Second line } \\
\text { drug: rituximab, } \\
\text { cyclophosphamide }\end{array}$ \\
\hline
\end{tabular}




\begin{tabular}{|c|c|c|c|c|}
\hline Limbic encephalitis ${ }^{7,8}$ & $\begin{array}{c}\text { Antibodies against } \\
\text { intraneuronal antigens: } \\
\text { Hu, Ma2, amphiphysin, } \\
\text { GAD } \\
\text { Antibodies against } \\
\text { synaptic antigens: } \\
\text { GABABR, mGluR5 }\end{array}$ & $\begin{array}{l}\text { Severe short-term } \\
\text { memory loss, } \\
\text { seizures }\end{array}$ & $\begin{array}{l}\text { EEG: temporal lobe } \\
\text { epileptic activity; slowing } \\
\text { MRI: increased T2 and } \\
\text { FLAIR signal in limbic } \\
\text { region } \\
\text { CSF: pleocytosis and } \\
\text { increased proteins }\end{array}$ & $\begin{array}{c}\text { Immunotherapy } \\
\text { Good response to cell } \\
\text { surface autoantigens }\end{array}$ \\
\hline \multicolumn{5}{|c|}{ Strongly suspected immune mechanism } \\
\hline $\begin{array}{l}\text { Opsoclonusmyoclonus and } \\
\text { other cerebellarbrainstem } \\
\text { encephalitis }\end{array}$ & $\begin{array}{l}\text { Anti } \mathrm{Hu} \text { ab found } \\
\text { sometimes } \\
\text { Found in } \\
\text { neuroblastoma and } \\
\text { teratoma }\end{array}$ & $\begin{array}{l}\text { Opsoclonus, irritability, } \\
\text { ataxia, } \\
\text { Fall, myoclonus, } \\
\text { Tremor }\end{array}$ & $\begin{array}{c}\text { CSF - B-cell } \\
\text { activation } \\
\text { MRI: cerebellar atrophy }\end{array}$ & $\begin{array}{l}\text { Immunotherapy } \\
\text { Mild response to } \\
\text { neuroblastoma } \\
\text { associated } \\
\text { Good response to } \\
\text { teratoma associated }\end{array}$ \\
\hline Bickerstaff encephalitis $^{10}$ & GQ1b antibodies & $\begin{array}{l}\text { Ophthalmoplegia, ataxia, } \\
\text { hyperreflexia. }\end{array}$ & $\begin{array}{c}\text { MRI: T2-signal } \\
\text { abnormalities in the } \\
\text { brainstem, thalamus, } \\
\text { and cerebellum) } \\
\text { NCS: predominant axonal } \\
\text { degeneration, } \\
\text { less frequent } \\
\text { demyelination }\end{array}$ & $\begin{array}{l}\text { good outcome } \\
\text { with steroids, IVIG } \\
\text { and/or plasma } \\
\text { exchange }\end{array}$ \\
\hline Hashimoto encephalitis ${ }^{11}$ & TPO antibodies & $\begin{array}{l}\text { Stroke-like symptoms, } \\
\text { tremor, myoclonus, } \\
\text { aphasia, seizures, ataxia }\end{array}$ & $\begin{array}{l}\text { EEG: slow activity } \\
\text { CSF: elevated protein }\end{array}$ & Steroid-responsive \\
\hline Rasmussen encephalitis $^{12}$ & $\begin{array}{l}\text { Most likely immune } \\
\text { mediated (unclear } \\
\text { mechanism) }\end{array}$ & $\begin{array}{l}\text { partial seizures, } \\
\text { cognitive decline, } \\
\text { focal deficits, } \\
\text { brain hemiatrophy }\end{array}$ & $\begin{array}{c}\text { MRI: progressive } \\
\text { unilateral hemispheric } \\
\text { atrophy }\end{array}$ & $\begin{array}{l}\text { Limited response to } \\
\text { Immunotherapy }\end{array}$ \\
\hline Basal ganglia encephalitis & $\begin{array}{c}\mathrm{D} 2 \mathrm{R} \text { ab in } \\
\text { some cases }\end{array}$ & $\begin{array}{c}\text { seizure } \\
\text { and behavior } \\
\text { disorder }\end{array}$ & $\begin{array}{l}\text { Variable basal ganglia } \\
\text { T2/FLAIR abnormalities }\end{array}$ & $\begin{array}{l}\text { Mostly monophasic, } \\
\text { can relapse }\end{array}$ \\
\hline \multicolumn{5}{|c|}{ Possible immune mechanism } \\
\hline $\begin{array}{l}\text { CLIPPERS: Chronic } \\
\text { lymphocytic inflammation } \\
\text { with pontine perivascular } \\
\text { enhancement responsive to } \\
\text { steroids }{ }^{13}\end{array}$ & Unknown & $\begin{array}{l}\text { Episodic diplopia, } \\
\text { symptoms of } \\
\text { brainstem and spinal } \\
\text { cord dysfunction }\end{array}$ & $\begin{array}{l}\text { MRI: enhancement } \\
\text { Of pons } \\
\text { and medulla, } \\
\text { brachium pontis, } \\
\text { cerebellum, midbrain, } \\
\text { and, occasionally, } \\
\text { spinal cord }\end{array}$ & Steroid responsive \\
\hline $\begin{array}{c}\text { ROHHAD: rapid onset } \\
\text { obesity with hypothalamic } \\
\text { dysfunction,hypoventilation } \\
\text { and autonomic } \\
\text { dysregulation }^{14}\end{array}$ & $\begin{array}{c}\text { Unknown } \\
\text { Associated with neural } \\
\text { crest tumors }\end{array}$ & $\begin{array}{l}\text { Rapid onset obesity, } \\
\text { hyperphagia, } \\
\text { abnormal behavior, }\end{array}$ & $\begin{array}{l}\text { MRI, usually } \\
\text { normal }\end{array}$ & $\begin{array}{l}\text { Limited response to } \\
\text { immunotherapy }\end{array}$ \\
\hline autonomic dysregulation $^{14}$ & & $\begin{array}{c}\text { autonomic } \\
\text { dysfunction, and } \\
\text { central } \\
\text { hypoventilation }\end{array}$ & & \\
\hline
\end{tabular}




\section{Management}

Despite the severity of many patients' symptoms, the majority of patients respond to treatment. Recovery can be slow and some disorders have a tendency to relapse. Corticosteroids and/ or intravenous immunoglobulins (IVIg) or plasma exchange are considered first-line therapies and should be considered in all patients. If the $\mathrm{AE}$ is associated with paraneoplastic picture then the primary tumor should be treated first. Patients who do not respond to the first-line therapies second-line immunotherapy with rituximab and/or cyclophosphamide should be started very soon [9-15].

\section{Conclusion}

$\mathrm{AE}$ is not so uncommon in children. They mostly mimic different psychiatric diseases. Most of the diseases are treatable. Steroid and other immunotherapy form primary treatment modality. Any child with features of unexplained psychosis, dementia, seizure, autonomic dysfunctions etc should be suspected for possibility of $\mathrm{AE}$ after ruling out other common causes. All neurophysicians as well as pediatricians must possess adequate knowledge about $\mathrm{AE}$ for early diagnosis and better management

\section{References}

1. Gable MS, Gavali S, Radner A Anti (2009) NMDA receptor encephalitis: report of ten cases and comparison with viral encephalitis. Eur J Clin Microbiol Infect 28(12): 1421-1429.

2. Lancaster E, Martinez Hernandez E, Dalmau J (2011) Encephalitis and antibodies to synaptic and neuronal cell surface proteins. Neurology 11(2): 179-189.

3. Armangue T, Petit Pedrol M, Dalmau J (2012) Autoimmune encephalitis in children. J Child Neurol 27(11): 1460-1469.

4. Kayser MS, Titulaer MJ, Gresa Arribas N, Dalmau J (2013) Frequency and characteristics of isolated psychiatric episodes in anti-N-methyl-daspartate receptor encephalitis. JAMA Neurol 70(9): 1133-1139.
5. Chapman MR, Vause HE (2011) Anti-NMDA receptor encephalitis: diagnosis, psychiatric presentation and treatment. Am J Psychiatry 168(3): 245-251.

6. Schmitt SE, Pargeon K, Frechette ES (2012) Extreme delta brush: a unique EEG pattern in adults with anti-NMDA receptor encephalitis. Neurology 79(11): 1094-1100.

7. Hoftberger R, Titulaer MJ, Sabater L(2013) Encephalitis and GABAB receptor antibodies: novel findings in a new case series of 20 patients. Neurology 81(17): 1500-1506.

8. Haberlandt E, Bast T, Ebner A (2011) Limbic encephalitis in children and adolescents. Arch Dis Child 96(2): 186-191.

9. Armangue T, Titulaer MJ, Sabater L (2014) A novel treatment-responsive encephalitis with frequent opsoclonus and teratoma. Ann Neurol 75: 850-856.

10. Odaka M, Yuki N, Yamada M (2003) Bickerstaff's brainstem encephalitis: clinical features of 62 cases and a subgroup associated with GuillainBarré syndrome. Brain 126: 2279-2290.

11. Dogan M, Acikgoz E, Acikgoz M (2011) The frequency of Hashimoto thyroiditis in children and the relationship between urinary iodine level and Hashimoto thyroiditis. J Pediatr Endocrinol Metab 24(1-2): 75-80.

12. Bien CG, Schramm J (2009) Treatment of Rasmussen encephalitis half a century after its initial description: promising prospects and a dilemma. Epilepsy Res 86(2-3): 101-112.

13. Pittock SJ, Debruyne J, Krecke KN (2010) Chronic lymphocytic inflammation with pontine perivascular enhancement responsive to steroids (CLIPPERS). Brain 133:2626-2634.

14. Ize-Ludlow D, Gray JA, Sperling MA (2007) Rapid-onset obesity with hypothalamic dysfunction, hypoventilation, and autonomic dysregulation presenting in childhood. Pediatrics 120(1): e179-e188.

15. Titulaer MJ, McCracken L, Gabilondo I (2013) Treatment and prognostic factors for long-term outcome in patients with anti-NMDA receptor encephalitis: an observational cohort study. Lancet Neurol 12: 157-165.

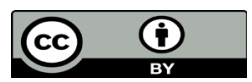

This work is licensed under Creative Commons Attribution 4.0 License

To Submit Your Article Click Here: Submit Article
DOI: $10.32474 /$ PAPN.2018.01.000109

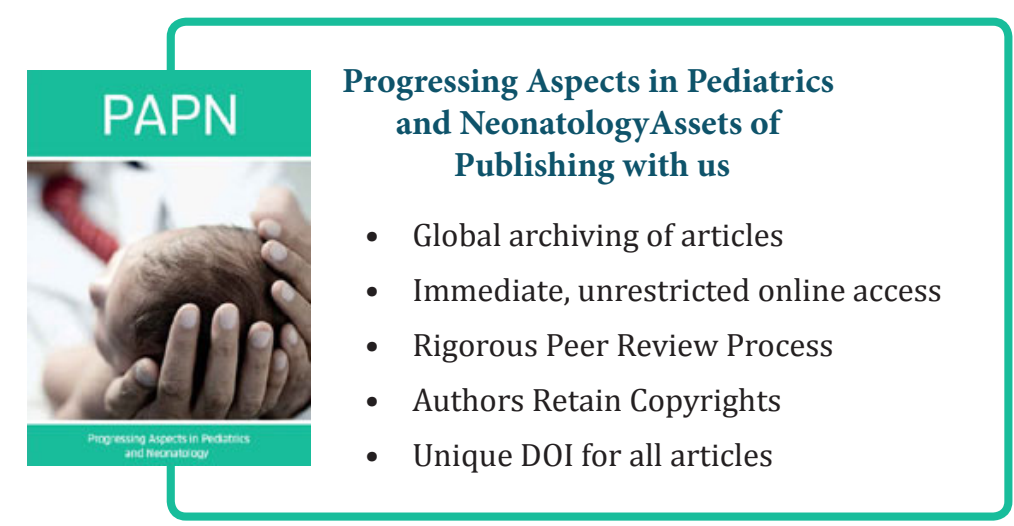

